\title{
Optical Coupling of Bare Optoelectronic Components and Flexographically Printed Polymer Waveguides in Planar Optronic Systems
}

\author{
Yixiao Wang*, Tim Wolfer, Alex Lange, Ludger Overmeyer \\ Institute of Transport and Automation Technology, Leibniz Universitaet Hannover, An der \\ Universitaet 2, 30823, Garbsen, Germany
}

\begin{abstract}
Large scale, planar optronic systems allowing spatially distributed functionalities can be well used in diverse sensor networks, such as for monitoring the environment by measuring various physical quantities in medicine or aeronautics. In these systems, mechanically flexible and optically transparent polymeric foils, e.g. polymethyl methacrylate (PMMA) and polyethylene terephthalate (PET), are employed as carrier materials. A benefit of using these materials is their low cost. The optical interconnections from light sources to light transmission structures in planar optronic systems occupy a pivotal position for the sensing functions. As light sources, we employ the optoelectronic components, such as edgeemitting laser diodes, in form of bare chips, since their extremely small structures facilitate a high integration compactness and ensure sufficient system flexibility. Flexographically printed polymer optical waveguides are deployed as light guiding structures for short-distance communication in planar optronic systems. Printing processes are utilized for this generation of waveguides to achieve a cost-efficient large scale and high-throughput production. In order to attain a high-functional optronic system for sensing applications, one of the most essential prerequisites is the high coupling efficiency between the light sources and the waveguides. Therefore, in this work, we focus on the multimode polymer waveguide with a parabolic cross-section and investigate its optical coupling with the bare laser diode. We establish the geometrical model of the alignment based on the previous works on the optodic bonding of bare laser diodes and the fabrication process of polymer waveguides with consideration of various parameters, such as the beam profile of the laser diode, the employed polymer properties of the waveguides as well as the carrier substrates etc. Accordingly, the optical coupling of the bare laser diodes and the polymer waveguides was simulated. Additionally, we demonstrate optical links by adopting the aforementioned processes used for defining the simulation. We verify the feasibility of the developed processes for planar optronic systems by using an active alignment and conduct discussions for further improvements of optical alignment.
\end{abstract}

Keywords: Optical coupling, optical simulation, optical alignment, transparent polymer foils, bonding of optoelectronic chips, flexographic printing, multimode polymer waveguides, planar optronic systems

\section{INTRODUCTION}

The perception of the world originates from the detection of diverse physical quantities of the environment, for which the use of sensors is indispensable. Nowadays, scientists and researchers are increasingly committed to designing innovative sensor concepts, developing and implementing sophisticated sensor modules and systems. Being distinguished from the conventional sensors based on electronics, optical sensors exhibit a greater potential in terms of high-speed data transfer and electromagnetic interference resistance. In addition to considering the performance, the manufacturing costs, as one of the most essential factors in the industry, must be taken into account as well. Roll-to-roll processes provide a highefficiency production, thus tremendously cutting down the cost of every production unit. In addition, material costs account for a large proportion and should therefore be substantially reduced as well. Thus, a promising proposal is to employ low cost polymeric foils with a thickness up to $200 \mu \mathrm{m}$ as carrier substrates of systems, which are mechanically flexible and optically transparent. The former ensures the compatibility with the roll-to-roll process and the latter enables a variety of optical sensing applications.

*yixiao.wang@ita.uni-hannover.de; phone +49 511762 18329; fax +49 511762 4007; www.ita.uni-hannover.de

Silicon Photonics and Photonic Integrated Circuits V, edited by Laurent Vivien, Lorenzo Pavesi, Stefano Pelli, Proc. of SPIE Vol. 9891, 989103 - (C) 2016 SPIE · CCC code: 0277-786X/16/\$18 · doi: 10.1117/12.2227340 
With this in mind, we propose to establish large scale sensor networks that are fully polymer-based, primarily with polymeric foils as carrier substrates [1]. In order to detect diverse signals and subsequently process and transfer them purely by optical means, a high density of optoelectronic and optical devices is to be integrated on or into the polymeric foils. In this way, a so-called planar optronic system is comprised, which allows numerous spatially distributed sensing functionalities. When extracting a transmission path from this highly integrated system, we can observe that the light is emitted by a light source, e.g. laser diode (LD) or light emitting diode (LED), transferred by a polymer optical waveguide, meanwhile utilized for sensing signals and finally sent to a detector, which is usually the photo diode. The key of the sensing principle is the utilization of the light properties, such as the intensity, phase or wavelength. Since these properties of light are sensitive to various physical quantities, diverse applications, such as the measurements of temperature, pressure or concentration of analytes are possible, especially in the field of medicine or aeronautics thanks to its immunity to the electromagnetic interference.

In order to ensure the sensing functionality of the planar optronic system, one of the most basic prerequisites is an adequate optical coupling efficiency from the light source to the waveguide. We employed polymethyl methacrylate (PMMA, Plexiglas XT 99524, ThyssenKrupp Plastics GmbH) with a thickness of $175 \mu \mathrm{m}$ as the carrier substrate of the planar optronic system. As the light source, we chose a bare edge-emitting laser diode (Roithner CHIP-650-P5) and accomplished its integration by applying the low temperature optodic bonding. The high throughput flexographic printing technology was adopted to fabricate a surface integrated multimode polymer waveguide (cf. Fig. 2a). We focused on the positioning accuracy of the laser diode and conducted geometry-based simulative investigations of its influences on the coupling efficiency with the waveguide. This can be used as positioning guidelines in the optodic bonding process. In addition, we fabricated optical links by adopting the aforementioned processes and investigated the issue of the optical alignment.

\section{MATERIALS}

As introduced, we employed a bare edge-emitting laser diode as the light source and fabricated multimode polymer waveguides as transmission structures. Here, we give a brief description of the important properties that are necessary to be confirmed for the simulative investigations.

\subsection{Bare laser diode}

The bare laser diode has a lasing wavelength of $655 \mathrm{~nm}$ (referred to as LD in following). It has a dimension of $300 \times 250 \times 100 \mu \mathrm{m}$ and emits light from both edges (cf. Fig. 1), which enables the butt coupling. The major advantage here is that no extra coupling elements, such as micro mirrors, are required.

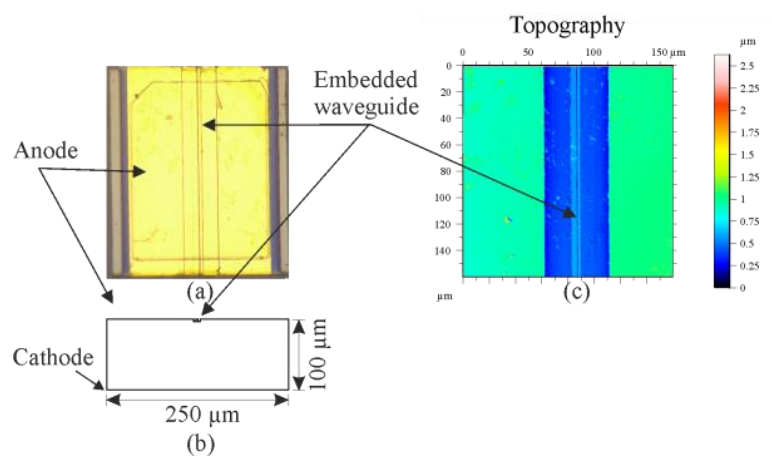

Figure 1. (a) Microscopic image of the upper face; (b) schematic side-view; (c) topography of the upper face of the laser diode CHIP-650-P5

As we can recognize in Fig. 1 (c), the waveguide in the LD lies along the longer edge of $300 \mu \mathrm{m}$ and is embedded in the middle of the surface with a ridge structure. Confocal microscopic measurements indicate that the ridge has a width of ca. $4 \mu \mathrm{m}$ and a depth of ca. $1.1 \mu \mathrm{m}$ from the top surface of the LD excluding the metallic layer. The side with the ridge waveguide is fully metalized and functions as the anode, while the opposite side functions as the cathode. It means that the anode of the LD is only ca. $1.1 \mu \mathrm{m}$ above the emitter, which is in the very close proximity of the emitter. 
According to the data sheet provided by the manufacturer, we obtained the beam divergence of the LD, which are typically 8 degrees in parallel direction and 32 degrees in perpendicular direction. The LD reaches its optical power of $5 \mathrm{~mW}$ by being driven under an operating current of $29 \mathrm{~mA}$.

\subsection{Polymer waveguides}

Flexographic printing is widely used for the purpose of visual printing where newspapers, brochures and packaging are produced. The application of this process to functional printing has been shown in the production of electrically conductive layers [2]. In the present work, the extension to the utilization of flexographic printing for printed optics is presented [3]. In this concept transparent fluid polymers are printed in multiple passes onto each other and thereby create a three dimensional structure. As waveguide core material, transparent acrylates in a liquid state with a dynamic viscosity of $200 \mathrm{mPas} @ 26^{\circ} \mathrm{C}$ are used (Jaenecke + Schneemann Druckfarben, UV Glanzlack praegefaehig). In the cured state, they show a refractive index of $1.516 @ 635 \mathrm{~nm}$. The underlying substrate acts as the waveguide's lower cladding and therefore needs to have a lower refractive index as the core. Because of this reason and the excellent available surface quality PMMA foils are employed. They show a refractive index of $1.49 @ 635 \mathrm{~nm}$ and therefore create a numerical aperture of the waveguide system of 0.28 .

\section{PROCESS TECHNOLOGIES}

\subsection{Optodic bonding for the surface integration of bare optoelectronic components}

The polymer foil PMMA attracts our attention to be chosen as the carrier substrate of planar optronic systems as it is very cost-efficient and optically transparent. However, we have to consider its low glass transition temperature (Tg) of $105^{\circ} \mathrm{C}$, which poses a big challenge of controlling the thermal loading while being processed. In order to comply with this, we developed a low temperature bonding technology, the so-called optodic bonding [4][5]. The key solution here is the utilization of the UV-irradiation as driving energy instead of the thermal effect to complete the bonding process. We adopted optodic bonding in a manual flip chip die bonding assembly system by designing a novel underplate to replace the conventional heat plate.

With the help of the assembly system, we can achieve a lateral positioning accuracy of $\pm 5 \mu \mathrm{m}$ in the $\mathrm{x}$ - and y-direction. In addition to this, a large portion of the positioning deviation derives from the tilt of the LD occurring during the bonding process. The most important influence on tilting is the pressure exerted on the LD. The inhomogeneous pressure distribution results in tilts of the LD around different rotating axis which can be described in the degree of tilt angles. Apart from that, the bondline thickness constitutes another important factor resulting from optodic bonding, which should be taken into account. Through a series of statistic measurements of the bonded samples by means of a confocal microscope, we estimated an approximate bondline thickness of $20 \mu \mathrm{m}$.

\subsection{Flexographic printing for the surface integration of multimode polymer waveguides}

The working principle of the flexographic printing process is based on three cylinders which rotate under a continuous contact pressure (cf. Fig. 2b). Fluid varnish is stored in an ink supply and fills cavities that are engraved on the surface of a rotating anilox roller. These cavities convey the varnish and subsequently wet embossed structures of the printing plate which is mounted onto the plate cylinder. These elevated structures in turn carry the fluid further and reproduce a mirrorinverted image of the printing plate on the printing substrate. The final curing by ultraviolet light causes a radical polymerization of the varnish.

Printed waveguides currently show a minimum width of $50 \mu \mathrm{m}$ and a height of $110 \mu \mathrm{m}$ (45 layers). The amount of layers determines the structures height. Each layer adds approx. $3.5 \mu \mathrm{m}$ in total height and a maximum of 45 layers has been stacked onto each other. At a total number of ten printing cycles per sheet a total throughput of 300-1,500 sheets/hour is generated with a sheet format of $350 \times 500 \mathrm{~mm}$. The throughput is dependent on the variable printing speed. Fig. 2c shows a printed polymer structure with a height of $34.6 \mu \mathrm{m}$ and a width of $235 \mu \mathrm{m}$. The surface exhibits a waviness Wa of $80 \mathrm{~nm}$ and a roughness $\mathrm{Ra}$ of $12.5 \mathrm{~nm}$. To examine the refractive index distribution of the printed structure a measurement by the refracted near-field method was performed along the vertical path in the z-direction as shown in Fig. 2c (refractive index profilometer, Rinck Elektronik, Germany). The refractive index shows a gradual progression between the individual cores and peripheral areas of the respective printed layers with a difference of $1.502 \cdot 10-3 \pm$ $2.33 \cdot 10-4$ between layer core and the associated boundary. 

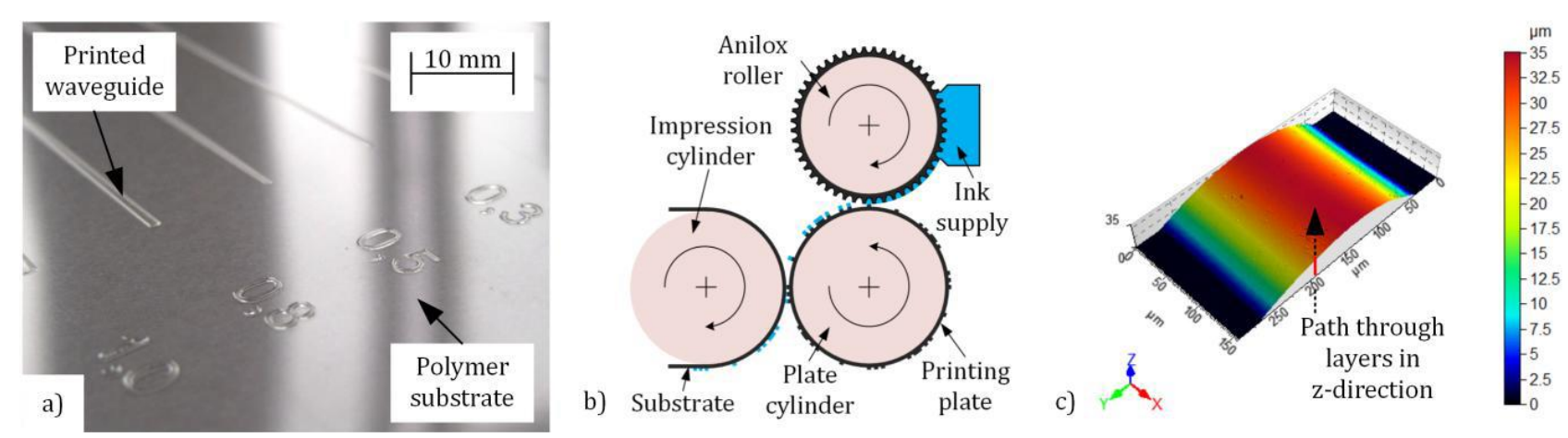

Figure 2. a) Flexographic printed waveguides (acrylate) on PVC substrate; b) Operating principle of flexographic printing process; c) Confocal microscopy of printed optical waveguide with indicated path for refractive index measurement.

\section{SIMULATION OF OPTICAL COUPLING}

In order to clarify the requirements of the alignment with the waveguide, which is mainly determined by the positioning of the LD during optodic bonding, we began the investigations with an optical simulation using the program Zemax. Here, we strive to figure out the influences of the positioning deviations on the efficiency of the optical coupling between the LD and the waveguide and thus to be able to define the tolerance range of the deviation. As described above, the total deviation comprises of the lateral part and the rotational part, which is expressed with a tilt angle in degrees. These two aspects were investigated in order to obtain the requirements of the positioning accuracy that should be achieved in the bonding process.

\subsection{Geometry of the alignment}

Determining the model of the alignment geometry between the LD and the waveguide is the first step to launch the simulation. It is shown in Fig. 3.

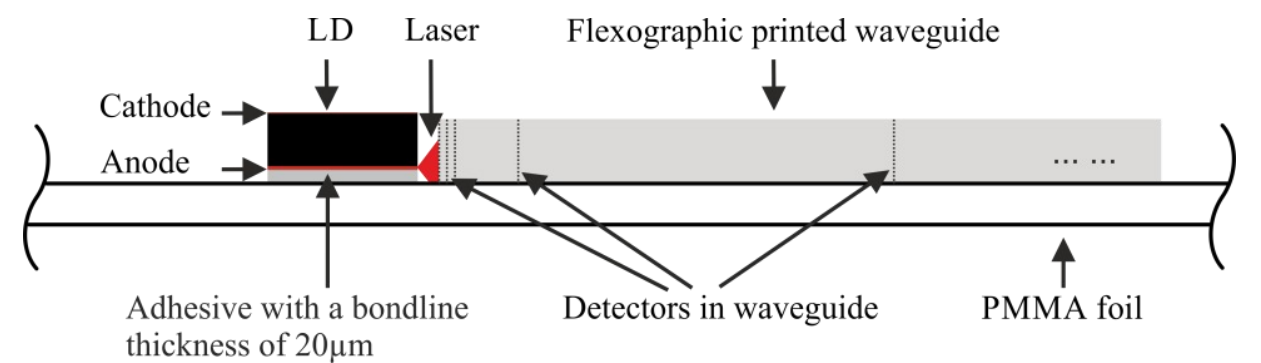

(a)

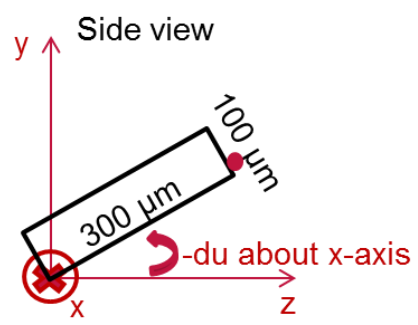

(b)

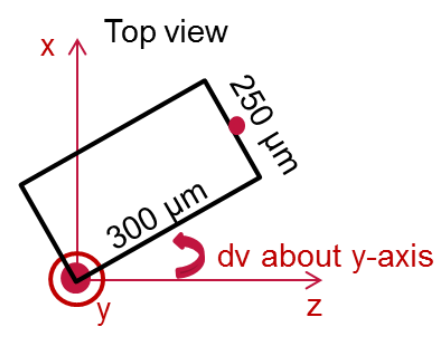

(c)

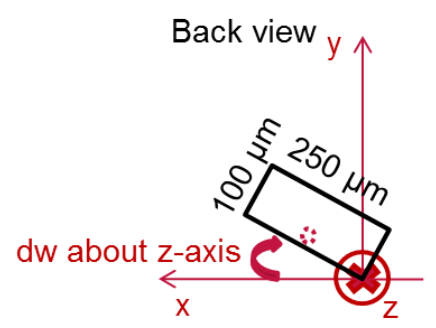

(d)

Figure 3. (a) Alignment geometry of the LD and the waveguide; (b) side view of the LD indicating the rotational deviation with the tilt du about $\mathrm{x}$-axis; (c) top view of the LD indicating the rotational deviation with the tilt dv about $y$-axis; (d) back view of the LD indicating the rotational deviation with the tilt dw about $\mathrm{z}$-axis;

Considering the issue of heat dissipation, we prefer to apply the bonding configuration of the LD with its emitter (episide) downwards in the simulation [6], because here the heat dissipation path can be shorter and the dissipation process 
can be accelerated. However, the realization of this configuration is quite complicated in terms of the fabrication process. As aforementioned, the emitter of the LD lies about $1.1 \mu \mathrm{m}$ lower from the top surface, which can be assumed being at the bottom of the LD when the LD is bonded epi-down. Since the bondline thickness is assumed to be $20 \mu \mathrm{m}$, the emitter of the LD is located about $21 \mu \mathrm{m}$ above the surface of the PMMA foil (cf. Fig. 3). The minimum achievable width of the flexographic printed waveguide is $50 \mu \mathrm{m}$ and we assume here to be $300 \mu \mathrm{m}$. Its length is assumed to be $200 \mathrm{~mm}$. The optical properties of the polymer waveguide made of transparent acrylates described above are considered and defined in the Zemax Simulation. The waveguide exhibits a parabolic form with a height of $110 \mu \mathrm{m}$, which means that its middle point has a distance of $55 \mu \mathrm{m}$ from the surface of the PMMA foil. In this case, the emitter of the LD is not aligned to the center of the waveguide, which will not result in an optimal optical coupling. However, we keep this initial alignment, which is directly adopted from the implementation of the fabrication technologies, in consideration of the convenience by saving extra treatments to achieve the optimal one. Another reason is that the LD has a relative big beam divergence of 32 degrees in perpendicular direction, which makes this initial alignment not that critical. Therefore, we started the investigation with this alignment and measured the coupled power at the spots with different distances from the LD by placing detectors at their positions in the middle axis of the waveguide. The resulting coupling efficiency was evaluated.

\subsection{Optical coupling}

In the Zemax simulation, we employed 10000 rays to emulate the total optical power of $5 \mathrm{~mW}$ and detected the transmitted power at different spots of the waveguide in reference of the distance from the LD (cf. Fig. 3 (a)). The influence of the positioning accuracy on the optical coupling was investigated by varying the lateral and rotational coordinate values of the LD.

\subsubsection{Influence of lateral deviation}

As aforementioned, we assumed the bondline thickness to be $20 \mu \mathrm{m}$ according to the confocal microscopy measurements. Thus, the coordinate y was assigned to be $20 \mu \mathrm{m}$. Since the applied die bonding assembly system provides a lateral positioning accuracy of $\pm 5 \mu \mathrm{m}$ and the alignment geometry is symmetric along the middle axis of the waveguide, we varied the coordinate $\mathrm{x}$ and $\mathrm{z}$ from 0 to $5 \mu \mathrm{m}$ both in steps of $0.2 \mu \mathrm{m}$. No rotational deviations occurred in this simulation (du, dv and dw were kept 0). Concerning the simulation results shown in Fig. 4, we can observe parallel planes representing the coupled power at different spots of the waveguide perpendicular to the axis representing the optical power, which means that little influence is caused for the optical coupling by the deviations of the lateral positioning within $5 \mu \mathrm{m}$. The power of the detector falls away from the LD according to the increase of its distance, which is in line with our expectation.

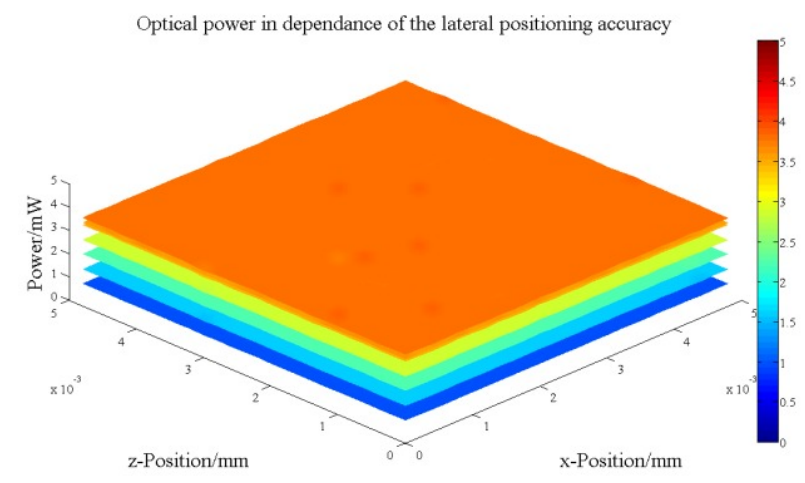

Figure 4. Coupled optical power detected at different spots of the waveguide with a positioning deviation within $5 \mu \mathrm{m}$ for the coordinate axis $\mathrm{x}$ and $\mathrm{z}$.

Hence, the positioning accuracy of the assembly system is adequate for bonding the LD of being optically appropriately aligned to the waveguide.

\subsubsection{Influence of rotational deviation}

In contrast to the lateral positioning accuracy mainly depending on the assembly system, the rotational deviation is essentially induced during the bonding process as a certain force is exerted on the bonding head while the adhesive is cured. An inhomogeneous pressure distribution results in an uneven curing mechanism and a tilting of the LD about a 
certain rotation axis. The direction of the laser emitter can be consequently changed, affecting the coupling efficiency with the waveguide. In order to classify the degree of influence of different rotation axis, we implemented simulations by varying the tilt degrees du, dv and dw separately (cf. Fig. 3 (b) (c) (d)). The lateral positioning was assumed ideally without deviations ( $\mathrm{x}$ and $\mathrm{z}$ were kept 0 ). According to the bonding geometry and the beam divergence of the LD with 8 degrees in parallel direction and 32 degrees in perpendicular direction specified by the manufacturer, we defined the range of the tilt variation for du between $0^{\circ}-30^{\circ}$, dv between $-15^{\circ}-15^{\circ}$ and $\mathrm{dw} 0^{\circ}-30^{\circ}$. The transmitted optical power detected at different spots of the waveguide in dependence of the tilt du and dv is illustrated in each diagram in Fig. 5, where the tilt $d w$ remains constant. With the increase of the tilt $d w$, its influence can be recognized from the gradual changes of the six diagrams in Fig. 5.

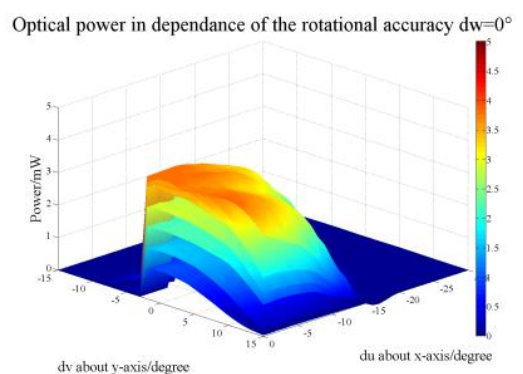

Optical power in dependance of the rotational accuracy $\mathrm{dw}=10^{\circ}$

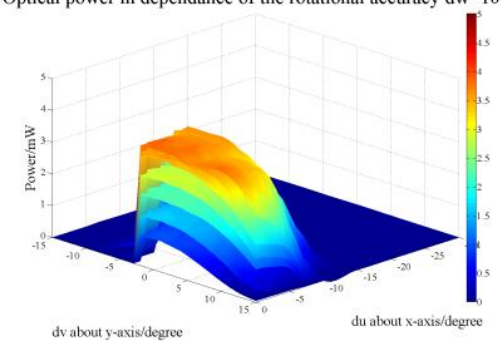

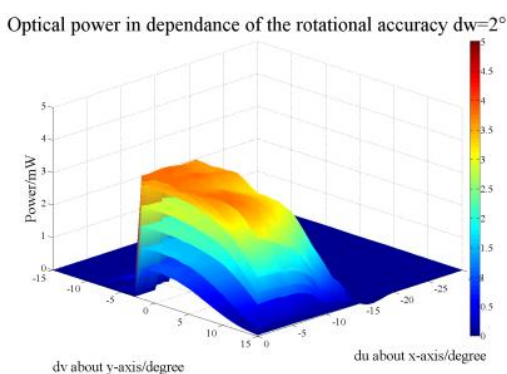

dv about $y$-axis/degre

Optical power in dependance of the rotational accuracy $\mathrm{dw}=20^{\circ}$

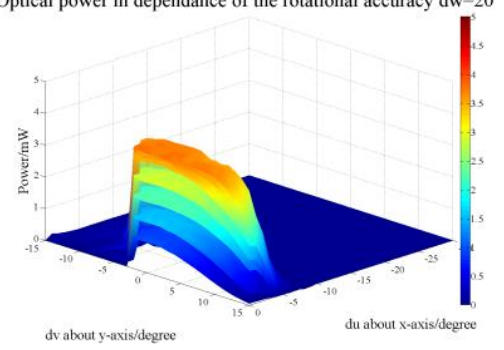

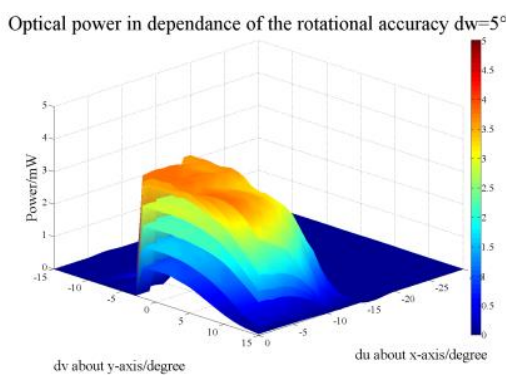

Optical power in dependance of the rotational accuracy $\mathrm{dw}=30^{\circ}$

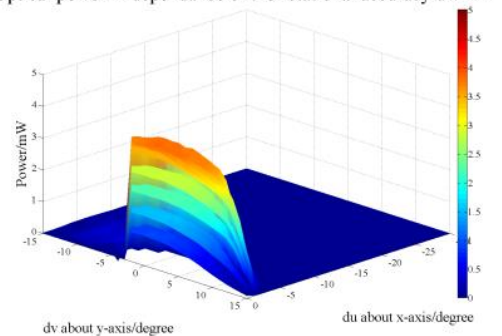

Figure 5. Coupled optical power detected at different spots of the waveguide in dependence of the tilt degrees about $\mathrm{x}, \mathrm{y}$ and $\mathrm{z}$ axis

The presented simulation results indicate that a change of the tilt $\mathrm{dv}$ about the y-axis within -2.5 and $2.5^{\circ}$ has little effect on the loss of optical power. However, the power decreases as the tilt increases. Worse is in the range between $-15^{\circ}$ to $7.5^{\circ}$, where the power is hardly coupled. For the tilt du about the $\mathrm{x}$-axis, the immunity range to the power loss narrows from $-3^{\circ}$ to $0^{\circ}$. A larger tilt in the range out of $-3^{\circ}$ causes a faster drop and a tilt of no coupled power will be reached as it keeps on growing. The determination of this tilt depends on the tilts about the other axes.

Observing the gradual changes between the diagrams with the increased tilt dw about the z-axis, a contraction of the range with an adequate optical power of $\mathrm{du}$ and $\mathrm{dv}$ can obviously be recognized. Ideally when no tilt dw occurs, light can still be coupled into the waveguide for the tilt $\mathrm{dv}$ with a range from $-5^{\circ}$ to $15^{\circ}$ and the tilt du from $-18^{\circ}$ to $0^{\circ}$. As the tilt $\mathrm{dw}$ grows to $30^{\circ}$, the range that can be optically coupled is significantly reduced to from $-5^{\circ}$ to $0^{\circ}$ for du, where the range of $d v$ remains. Nevertheless, as exhibited in the diagrams, the tilt $d w$ hardly influences the optical coupling power when it is kept within $5^{\circ}$.

With these simulation results we can define the tolerance range of the tilt deviations for a certain coupling efficiency that is to be achieved. They can be used as guidelines for the bonding process in order to attain an adequate optical alignment with the waveguide. The basic rule for a little loss of optical coupling, which can be summarized from the results above is the restriction of the tilt from $-3^{\circ}$ to $0^{\circ}$ about $\mathrm{x}$-axis, from $-2.5^{\circ}$ to $2.5^{\circ}$ about $\mathrm{y}$-axis and from $0^{\circ}$ and $5^{\circ}$ about $\mathrm{z}$-axis. This is based on the geometry of the optimal alignment in Fig. 3(a). It implies a more tolerant range of deviations for the bonding process in case of an optimal alignment.

\section{OPTICAL COUPLING USING ACTIVE ALIGNMENT}

In addition to the simulation, we also realized the optical links by applying optodic bonding to integrate the bare edgeemitting laser diode and flexographic printing to fabricate the polymer multimode waveguide onto the PMMA foil. Considering the convenience of the fabrication processes, the bonding of the LD and the printing of the waveguide were 
completed separately on two identical PMMA foils. Both components are to be positioned on the edge of each foil to cope with the alignment in terms of the geometry of a butt coupling (cf. Fig. 3 (a)). An additional carrier substrate was employed to support the LD and the waveguide composing an optical link (Fig. 6).

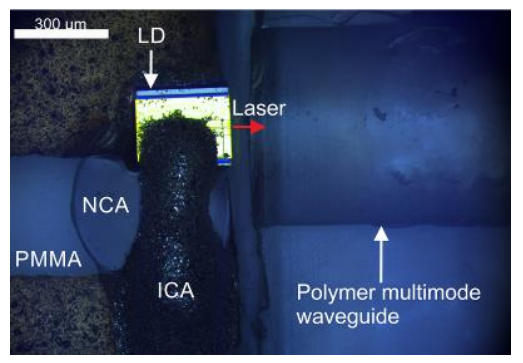

Figure 6. Microscopic image of the optical link consisting of optodic bonded bare edge-emitting LD and flexographic printed polymer multimode waveguide.

The LD shown in Fig. 6 was bonded with the epi-side upwards. The main reason here is the ultra-closeness of the emitter (epi-side) to the anode of the LD $(1.1 \mu \mathrm{m})$, which complicates the implementation of the bonding process with the anode downwards (epi-side downwards), since the emitter should not be covered by the applied adhesive. Thus, due to the convenient control of the process, we focused more on the bonding configuration with the epi-side upwards. Nevertheless, we also fabricated working optical links with the epi-side downwards to conform to the simulation and verify the simulated results. However, in both bonding configurations, a high-efficiency optical coupling could hardly be achieved when applying a conventional passive alignment since the bondline thickness cannot be determined accurately despite of the estimated range received from statistic measurements. Due to this fact, we decided to carry out the active alignment [7] using an optical measurement stage, in which the LD is fixed and the waveguide can be moved. The optical coupling efficiency between the LD and the waveguide can be investigated by illustrating the intensity distribution observed from the end facet of the waveguide.

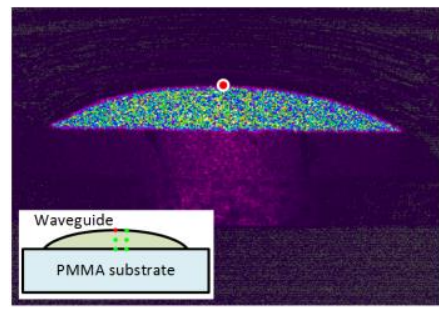

a)

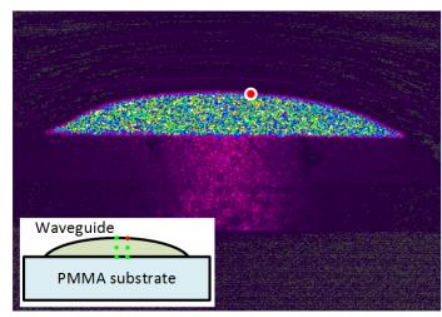

d)

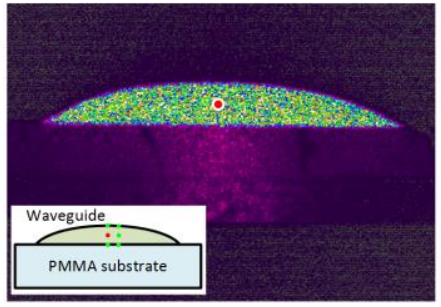

b)

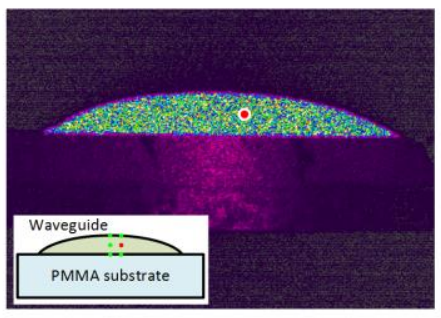

e)

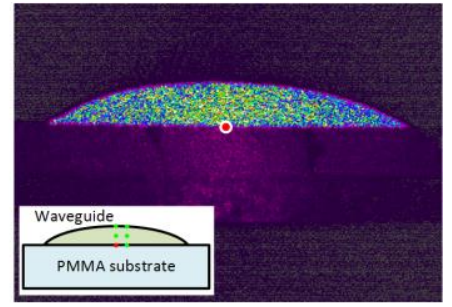

c)

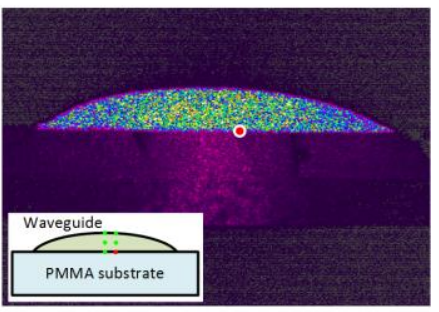

f)

Figure 7. Intensity distributions of the coupled light from the LD (center position indicated by red dot) observed from the end facet of the waveguide: (a) waveguide $50 \mu \mathrm{m}$ lower aligned to the LD emitter; (b) waveguide optimal aligned to the LD emitter; (c) waveguide $50 \mu \mathrm{m}$ higher aligned to the LD emitter; (d) waveguide $50 \mu \mathrm{m}$ lower and left aligned to the LD emitter; (e) waveguide $50 \mu \mathrm{m}$ left aligned to the LD emitter; (f) waveguide $50 \mu \mathrm{m}$ higher and left aligned to the LD emitter.

Fig. 7 shows that most of the light is coupled into the waveguide while a small proportion is coupled into the substrate, which, however, is the loss of the transmission. This effective optical coupling obtained by the active alignment verifies the feasibility of the developed processes, the optodic bonding and the flexographic printing for the fabrication of optical links in planar optronic systems. The light is basically distributed homogenously in the waveguide. Through a fine 
adjustment of the waveguide, the optimal alignment (b) was attained according to the criteria with a maximum total coupling intensity. For this optimal alignment, we assumed that the position, to which the LD emitter is aligned, lies in the middle of the waveguide. In this way, the optimal position aligned to the LD emitter was determined and can be used to indicate the misalignment. By deviating the waveguide away from this optimal position, the influence of the misalignment is revealed. Since the optical link is symmetric, only the waveguide's half is to be investigated. The intensity distributions at the misaligned positions with $50 \mu \mathrm{m}$ up- and downwards as well as $50 \mu \mathrm{m}$ to the left with additionally $50 \mu \mathrm{m}$ up- and downwards from the optimal alignment displayed in Fig. 7 correspond to the coupled light from the LD into the waveguide and also imply the coupling efficiencies. The intensity at the misalignment with $50 \mu \mathrm{m}$ to the left (e) is slightly weaker than the one being optimally aligned, however it is appreciably stronger than the misaligned one with the same deviation of $50 \mu \mathrm{m}$ up- or downwards (a, c). Logically, a worse coupling intensity is expected at the misalignments with more deviations $(\mathrm{d}, \mathrm{f})$.

\section{CONCLUSION AND OUTLOOK}

Large scale polymer-based planar optronic systems applied as sensor networks consist of various transmission paths, where the efficient optical coupling between the light sources/detectors and the waveguides is one of the most challenging issues affecting the sensing functionalities. Concerning the fabrication of such systems, conventional technologies are not able to fulfill all requirements and conform to the compatibility. Hence, novel processes are necessary. To comply with the low Tg of polymer foils, low temperature optodic bonding was developed and applied to integrate the bare edge-emitting laser diode as light source onto the PMMA foil. For a cost-efficient, yet high-throughput fabrication of the waveguides onto the large scale PMMA foil, flexographic printing is employed. With focus on the investigations of the optical coupling, we conducted simulations using the program Zemax by establishing a geometry model based on both processes. The results in terms of the lateral and rotational deviations provide comprehensive guidelines for the positioning in optodic bonding, which indicate little influence of the lateral deviations within $5 \mu \mathrm{m}$ and of rotational deviations about $\mathrm{x}$-axis from $-3^{\circ}$ to $0^{\circ}$, y-axis from $-2.5^{\circ}$ to $2.5^{\circ}$ and z-axis from $0^{\circ}$ to $5^{\circ}$. In addition, we demonstrated fabricated optical links by adopting the introduced processes. However, the utilization of the passive alignment based on the geometry model is not adequate for achieving a sufficient optical coupling between the laser diode and the waveguide. Therefore, we applied active alignment to achieve an optimal alignment by illustrating intensity distributions displayed at the end facet of the waveguide. Thus, we confirmed the effective optical coupling between the optodic bonded laser diode and the flexographic printed waveguide and thus verified the feasibility of both processes for the fabrication of the optical links applicable to planar optronic systems.

Active alignment provides a precise coupling accuracy. However, it also needs a high adjustment effort, which is usually used for unknown laser sources or alignments with extremely high requirements of the accuracy for under submicron, e.g. alignment with singlemode waveguides. In case of multimode waveguides, a passive alignment shows a greater potential. Given this, we are developing approaches based on passive alignment and further self-alignment. Here, the process influences on the geometry and the 3D-structure of the fabricated samples must be investigated. In addition, measuring methods and technologies enabling effective and precise 3D-measurements play an essential role for the implementation of the passive alignment and thus should be further developed and improved.

\section{ACKNOWLEDGEMENT}

We gratefully acknowledge financial support from the Deutsche Forschungsgemeinschaft (DFG) within the framework of the Collaborative Research Center Transregio 123 - Planar Optronic Systems (PlanOS).

\section{REFERENCES}

[1] Collaborative research center Planar Optronic Systems (PlanOS), Available: http://www.planos.uni-hannover.de

[2] Deganello, D., Cherry, J.A., Gethin, D.T., Claypole, T.C., 2012, Impact of metered ink volume on reel-to-reel flexographic printed conductive networks for en-hanced thin film conductivity, Thin Solid Films 520 (6), 22332237. 
[3] Wolfer T. et al. Printing and preparation of integrated optical waveguides for optronic sensor networks. Mechatronics (2015), http://dx.doi.org/10.1016/j.mechatronics.2015.05.004

[4] Y. Wang, L. Overmeyer, "Low temperature optodic bonding for integration of micro optoelectronic components in polymer optronic systems," in Proc. 2nd International Conference on System-integrated Intelligence: Challenges for Product and Production Engineering (SysInt), Bremen, Jul 2-4, 2014, pp. 547-556

[5] Y. Wang, M. Akin, L. Jogschies, L. Overmeyer, L. Rissing, "Optodic Bonding of Optoelectronic Components in Transparent Polymer Substrates-Based Flexible Circuit Systems," in Proc. SPIE Photonics West OPTO, San Francisco, Feb 7-12, 2015.

[6] Teo Jin Wah Ronnie, "Advances in High-Power Laser Diode Packaging, Semiconductor Laser Diode Technology and Applications," Dr. Dnyaneshwar Shaligram Patil (Ed.), Croatia, 2012, pp. 321-340, ISBN: 978-953-51-0549-7, InTech, Available from: http://www.intechopen.com/books/semiconductor-laser-diodetechnology-andapplications/advances-in-high-power-laser-diode-packaging

[7] Sing H. Lee, Y.C. Lee, "Optoelectronic Packaging for Optical Interconnects," OPN, pp. 40-45, January 2006 